\title{
28 Research Soure

\section{Usefulness of Palliative Radiotherapy in Reducing the Frequency of Red Blood Cell Transfusion in Patients with Malignant Tumor Bleeding}

Atsuto Katano ( $\sim$ katanoa-rad@h.u-tokyo.ac.jp )

University of Tokyo Hospital

Hideomi Yamashita

University of Tokyo Hospital

\section{Research Article}

Keywords: red blood cell transfusion, hemostatic radiotherapy, palliative care

Posted Date: July 27th, 2021

DOl: https://doi.org/10.21203/rs.3.rs-690242/v1

License: (c) (1) This work is licensed under a Creative Commons Attribution 4.0 International License.

Read Full License 


\section{Abstract}

Purpose: We aimed to evaluate the usefulness of palliative radiotherapy in reducing the frequency of red blood cell (RBC) transfusions in patients with malignant tumor bleeding as a quantitative value to evaluate the hemostatic effect.

Patients and methods: In this study, we conducted a retrospective review of patients visiting our department between June 2013 and April 2021. All patients underwent RBC transfusions for 30 days before the initiation of radiotherapy. We determined the overall survival time and RBC transfusion-free time using statistical tests.

Results: In total, 21 patients were enrolled in our study, including 13 women and 8 men; the median patient age was 63 years (range: 32 to 82 years). After radiotherapy, 19 of the 21 patients had a decreased frequency of requirement for RBC transfusion compared to that before radiotherapy. Fourteen patients had achieved an RBC transfusion-free state one month after radiotherapy. The estimated 1-year overall survival and RBC transfusion-free rates were 36.3\% (95\% confidence interval [CI]: $14.2 \%$ ) and $66.7 \%$ (95\% Cl: $42.5 \%-42.5 \%)$, respectively.

Conclusion: The present study revealed a reduction in RBC transfusion after palliative radiotherapy in patients with malignant tumor bleeding. We believe that reducing the frequency of blood transfusions in patients with terminal conditions would have significant clinical benefits.

\section{Introduction}

The incidence of clinically significant tumor bleeding in patients with advanced-stage cancer is approximately $6 \%-14 \%$ (1). Prolonged tumor bleeding results in progressive anemia, which causes fatigue, dyspnea, shortness of breath, dizziness, and lightheadedness. Red blood cell (RBC) transfusion directly improves anemia and is a mainstay in the treatment of patients with general anemia(2). For symptom palliation in patients with advanced cancer, RBC transfusion has the potential to relieve symptoms such as fatigue and dyspnea(3). However, for patients in the terminally ill state, the impact of anemia could be further exacerbated by other underlying comorbidities, nutritional deficiencies, dietary intake, reduced functional status, and insufficient environmental support(4). Therefore, the threshold for $\mathrm{RBC}$ transfusion used in the general patient population is not necessarily applicable to terminal care population(5). There is an urgent need to clarify the benefits and risks of RBC transfusion in the terminal care state(6).

Since the discovery of X-rays, palliative radiation therapy has been used to relieve the symptoms of cancer for more than 100 years (7). Recent accumulating data suggest that the aggressive use of highconformal therapy, including stereotactic ablative radiotherapy, in patients with limited metastases can not only improve symptoms but also increase disease-free and overall survival rates $(8,9)$. These new directions are very promising, but the conventional usage of these therapies continues to be in high demand and requires a high level of skill and expertise. 
The hemostatic effect on tumor bleeding is one of the main clinical benefit of palliative radiotherapy. Several retrospective studies have revealed the high efficacy of hemostatic radiotherapy $(10,11)$; however, there is no consensus on the criteria for evaluating the hemostatic effect.

The present study focused on the usefulness of palliative radiotherapy in reducing the frequency of RBC transfusion in patients with malignant tumor bleeding as a quantitative value to evaluate the hemostatic effect.

\section{Material And Methods}

This study was conducted through a retrospective review of patients visiting our department between June 2013 and April 2021. The inclusion criteria were as follows: (i) referral to our department for palliative intent of malignant tumor bleeding, (ii) RBC transfusion requirements for 30 days before the initiation of radiotherapy, and (iii) sufficient data in medical records for assessment. Patients who lost to follow after radiotherapy only within one month were excluded.

All procedures performed in the studies involving human participants were in accordance with the ethical standards of the Declaration of Helsinki.

The study procedures were approved by our institutional review board (the Research Ethics Committee of the University of Tokyo) and written informed consent was obtained from all patients.

The present study focused on the reduction in the frequency of RBC transfusion by palliative radiotherapy in patients with malignant tumor bleeding. "pre-RT-RBC" was defined as transfusion of the required units of RBC for 30 days before the initiation of radiotherapy. "post-RT-RBC" was defined as transfusion of the required units of RBC for 30 days after the completion of radiotherapy. One unit of RBC transfusion had nominal volumes of $140 \mathrm{~mL}$, which was derived from $200 \mathrm{~mL}$ of whole blood.

The statistical software program $\mathrm{R}$ was used for statistical computing and graphics. Statistical significance was set at $p<0.05$. Overall survival (OS) time was defined as the time from the start of radiotherapy to the date of death from any cause. RBC transfusion-free (RBCTF) time was defined as the time from the end of radiotherapy to the date of transfusion, wherein patients who died from any cause were treated as censored cases. Wilcoxon signed-rank test was used to compare the two matched samples.

\section{Results}

The patient characteristics are described in Table 1. A total of 21 patients were enrolled in our study, including 13 women and 8 men, with a median age of 63 years, ranging from 32 to 82 years. For most patients, the performance status was poor at the time of palliative radiotherapy, and the median Karnofsky Performance Status was 70\%. The most frequent primary disease was gynecological cancer, followed by gastrointestinal and urological cancers. The other primary diseases were melanoma and 
angiosarcoma. The most frequent pathological type was adenocarcinoma, including endometrioid carcinoma, followed by squamous cell carcinoma. The median pre-RT-RBC value was 6 units, ranging from 2 to 30 . The median serum hemoglobin concentration before radiotherapy was $9.6 \mathrm{~g} / \mathrm{dL}$, ranging from 4.6 to $11.1 \mathrm{~g} / \mathrm{dL}$.

Table 1

Baseline characteristics of the 21 patients who underwent radiotherapy in our study

\begin{tabular}{|c|c|c|}
\hline Characteristics & & Number (percent) \\
\hline \multirow[t]{2}{*}{ Sex } & Male & $8(38 \%)$ \\
\hline & Female & $13(62 \%)$ \\
\hline Age & Median (range) & $63(32-82)$ \\
\hline \multirow[t]{5}{*}{ Karnofsky Performance Status } & $90 \%$ & $4(19 \%)$ \\
\hline & $80 \%$ & $8(38 \%)$ \\
\hline & $70 \%$ & $5(24 \%)$ \\
\hline & $60 \%$ & $3(14 \%)$ \\
\hline & $50 \%$ & $1(5 \%)$ \\
\hline \multirow[t]{4}{*}{ Primary disease } & Gynecological cancer & $10(48 \%)$ \\
\hline & Gastrointestinal cancer & $6(29 \%)$ \\
\hline & Urological cancer & $3(14 \%)$ \\
\hline & Others & $2(10 \%)$ \\
\hline \multirow[t]{3}{*}{ Pathological type } & Adenocarcinoma & $14(67 \%)$ \\
\hline & Squamous cell carcinoma & $4(19 \%)$ \\
\hline & Others & $3(14 \%)$ \\
\hline \multirow[t]{2}{*}{ History of previous radiotherapy } & Yes & $5(24 \%)$ \\
\hline & No & $16(76 \%)$ \\
\hline Red blood cell transfusion before RT & Median (range) [Unit] & $6(2-30)$ \\
\hline
\end{tabular}

The treatment schedule for radiotherapy is shown in Table 2. More than half of the patients were treated with $30 \mathrm{~Gy}$ in 10 fractions. After radiotherapy, 19 of the 21 patients (90.5\%) showed a decrease in post-RTRBC scores compared to that pre-RT-RBC. For the entire cohort, post-RT-RBC was significantly decreased $(p<0.001)$, with a median value of 0 units, ranging from 0 to 6 . Figure 1 presents a waterfall plot that shows the change in pre-RT-RBC to post-RT-RBC for each patient. Fourteen patients (66.7\%) had achieved an RBC transfusion-free state for 30 days after the end of radiotherapy. The median serum hemoglobin 
concentration after radiotherapy was $9.7 \mathrm{~g} / \mathrm{dL}$ ranging from 6.7 to $10.7 \mathrm{~g} / \mathrm{dL}$, which does not have any statistically significant difference before radiotherapy $(p=0.153)$.

Table 2

Radiotherapy dose-fractionation schedules in our study

\begin{tabular}{|llll|}
\hline Treatment schedule & Number (percent) & N & age \\
\hline 30 Gy in 10 fractions & $14(67 \%)$ & 14 & $(67)$ \\
\hline 20 Gy in 5 fractions & $3(14 \%)$ & 3 & $(14)$ \\
\hline 15 Gy in 3 fractions & $1(5 \%)$ & 1 & $(5)$ \\
\hline 8 Gy in 1 fraction & $1(5 \%)$ & 1 & $(5)$ \\
\hline 8 Gy in 2 fractions & $2(10 \%)$ & 2 & $(10)$ \\
\hline
\end{tabular}

The median follow-up period for all the patients was 3.5 months, ranging from 1.1 to 37.7 months. The median overall survival time was 6.4 months ( $95 \%$ confidence interval $[\mathrm{Cl}]: 1.8-37.5)$ months, and the estimated 1-year OS and RBCTF rates were $36.3 \%$ (95\% Cl: $14.2-59.2 \%)$ and $66.7 \%$ (95\% Cl: $42.5-36.5 \%)$, respectively (Fig. 2,3). No severe radiation-induced adverse events were observed during radiotherapy.

\section{Discussion}

This study revealed a significant improvement in the required number of RBC transfusions between preand post-radiotherapy. Repeated blood transfusions can result in post-transfusion iron overload syndrome, which causes iron-related toxicity as well as multiple organ damage due to iron deposition in tissues(12). A greater number of transfused units is also a risk factor for febrile non-hemolytic transfusion reaction, which is an immune reaction of recipient antibodies reacting to donor-derived antigens (13). Moreover, transfusion itself imposes a load on the respiratory and circulatory systems and causes transfusion-related acute lung injury and/or transfusion-associated circulatory overload (14). In clinical practice, Sirianni et al. reported that transfusions in palliative care units were infrequent because of the lack of definitive evidence about its utility (15). Considering this background, we believe that reducing the frequency of blood transfusions would be of great benefit to patients in palliative care.

Surgical intervention to remove bleeding tissues or ligate blood vessels is the most direct method of treatment for tumor bleeding. However, this form of intervention is often technically difficult due to the abnormal structure of the tumor and the general condition of the patient being not good enough to withstand surgery. Therefore, careful patient selection is essential (16). Krouse et al. insisted that patients and their families who underwent surgical intervention in the palliative care unit should be aware of the risks of high morbidity and mortality (17).

Trans-arterial embolization, the intentional blocking of arteries with small particles or mechanical devices, is another treatment modality used as a palliative treatment to reduce bleeding. It is also used as a palliative treatment to reduce pain associated with symptomatic bone metastases (18). Although the 
embolization procedure has been used to treat bleeding from many tumor sites with excellent results, there are potential limiting factors such as the inability to access the vessel of the tumor with a catheter owing to its location and degree of invasion $(19,20)$. There are also risks such as damage to peripheral organs and the possibility of ischemic organ damage. Post-embolization syndrome is characterized by fever, nausea and vomiting, and flu-like symptoms caused by an immune response to necrotic tissue, which may last for several days after embolization(21). Therefore, patients should be provided with adequate explanation about this procedure by an interventional radiology specialist.

Endoscopic treatment has proven to be effective for bleeding from the gastrointestinal tract, lungs, and bladder(22, 23). This method has the advantage of being able to see the bleeding site directly, allowing for both diagnostic biopsy and therapeutic intervention. The bleeding site can be treated by clipping, thermal cauterization, laser coagulation, or topical application of thrombin or fibrinogen(24). This technique targets only organs that can be reached endoscopically and is associated with endoscopic pain and distress with only mild sedation.

Systemic agents such as octreotide, vitamin K, vasopressin, tranexamic acid, and aminocaproic acid are used in the treatment of malignant bleeding(25). In patients with poor performance status, these drug administrations are less invasive procedures; however, their efficacy is very limited(26). Complementary procedures, such as discontinuation of anticoagulants and antiplatelet agents, may also be employed.

The present study has some limitations. The primary limitation is the small sample size, which might affect the reliability of the results, with a higher risk of several types of bias. The second limitation is the treatment heterogeneity in the radiotherapy schedule, such as total dose and fractionation. Third, our results were obtained based on the information present in the medical records of our hospital, which might have been imperfect to yield detailed information about the patients, such as physical condition and comorbidity.

In conclusion, the present study revealed a reduction in the frequency of RBC transfusion after palliative radiotherapy in patients with malignant tumor bleeding. We believe that reducing the number of blood transfusions in patients with terminal conditions would have significant clinical benefits. Therefore, future studies on this topic are warranted to elucidate the efficacy and safety of palliative radiotherapy.

\section{Declarations}

Funding: This work was supported by the Japan Society for the Promotion of Science (JSPS) KAKENHI Grant Number 21K15639.

Conflicts of interest/Competing interests: The author reports no conflicts of interest in this work.

Availability of data and material: The data that support the findings of this study are available from the corresponding author, upon reasonable request. 
Authors' contributions: AK wrote the draft and conducted statistical analyses. AK and HY collected and interpreted the clinical data. HY conceived the present study and participated in its design and coordination. All authors have revised this report and approved the final manuscript.

Ethics approval: All study procedures were approved by the Research Ethics Committee of the University of Tokyo.

\section{References}

1. Harris DG and Noble SI: Management of terminal hemorrhage in patients with advanced cancer: a systematic literature review. J Pain Symptom Manage 38: 913-927, 2009.

2. Cortés Buelvas A: Anemia and transfusion of red blood cells. Colomb Med (Cali) 44: 236-242, 2013.

3. Preston NJ, Hurlow A, Brine $\mathrm{J}$ and Bennett MI: Blood transfusions for anaemia in patients with advanced cancer. Cochrane Database Syst Rev: CD009007, 2012.

4. Martinsson $\mathrm{U}$ and Lundström S: The use of blood transfusions and erythropoietin-stimulating agents in Swedish palliative care. Support Care Cancer 17: 199-203, 2009.

5. Raval JS and Cooling LL: Red blood cell transfusion in palliative care: what are we doing and why are we doing it? Transfusion 58: 3-4, 2018.

6. Chin-Yee N, Taylor J, Rourke K, et al.: Red blood cell transfusion in adult palliative care: a systematic review. Transfusion 58: 233-241, 2018.

7. Lutz ST: Palliative radiotherapy: history, recent advances, and future directions. Ann Palliat Med 8: 240-245, 2019.

8. Palma DA, Olson R, Harrow S, et al.: Stereotactic ablative radiotherapy versus standard of care palliative treatment in patients with oligometastatic cancers (SABR-COMET): a randomised, phase 2, open-label trial. Lancet 393: 2051-2058, 2019.

9. Palma DA, Olson R, Harrow S, et al.: Stereotactic Ablative Radiotherapy for the Comprehensive Treatment of Oligometastatic Cancers: Long-Term Results of the SABR-COMET Phase II Randomized Trial. J Clin Oncol 38: 2830-2838, 2020.

10. Hughes $C$ and Radhakrishna $G$ : Haemostatic radiotherapy for bleeding cancers of the upper gastrointestinal tract. Br J Hosp Med (Lond) 80: 579-583, 2019.

11. Yu J, Jung J, Park SR, et al.: Role of palliative radiotherapy in bleeding control in patients with unresectable advanced gastric cancer. BMC Cancer 21: 413, 2021.

12. Shander A, Cappellini MD and Goodnough LT: Iron overload and toxicity: the hidden risk of multiple blood transfusions. Vox Sang 97: 185-197, 2009.

13. Menis M, Forshee RA, Anderson SA, et al.: Febrile non-haemolytic transfusion reaction occurrence and potential risk factors among the U.S. elderly transfused in the inpatient setting, as recorded in Medicare databases during 2011-2012. Vox Sang 108: 251-261, 2015. 
14. Roubinian N: TACO and TRALI: biology, risk factors, and prevention strategies. Hematology Am Soc Hematol Educ Program 2018: 585-594, 2018.

15. Sirianni G, Perri G, Callum J, Gardner S, Berall A and Selby D: A Retrospective Chart Review of Transfusion Practices in the Palliative Care Unit Setting. Am J Hosp Palliat Care 36: 185-190, 2019.

16. Blakely AM, McPhillips $\mathrm{J}$ and Miner TJ: Surgical palliation for malignant disease requiring locoregional control. Ann Palliat Med 4: 48-53, 2015.

17. Krouse RS, Nelson RA, Farrell BR, et al.: Surgical palliation at a cancer center: incidence and outcomes. Arch Surg 136: 773-778, 2001.

18. Koike Y, Takizawa K, Ogawa Y, et al.: Transcatheter arterial chemoembolization (TACE) or embolization (TAE) for symptomatic bone metastases as a palliative treatment. Cardiovasc Intervent Radiol 34: 793-801, 2011.

19. Lee SM, Jeong SY, Shin JH, et al.: Transcatheter arterial embolization for gastrointestinal bleeding related to pancreatic adenocarcinoma: clinical efficacy and predictors of clinical outcome. Eur $\mathrm{J}$ Radiol 123: 108787, 2020.

20. Kwon JH and Han YH: Efficacy and safety of superselective trans-catheter arterial embolization of upper and lower gastrointestinal bleeding using N-butyl-2-cyanoacrylate. Emerg Radiol 25: 111-120, 2018.

21. Arslan M and Degirmencioglu S: Risk Factors for Postembolization Syndrome After Transcatheter Arterial Chemoembolization. Curr Med Imaging Rev 15: 380-385, 2019.

22. Kim YI, Choi IJ, Cho SJ, et al.: Outcome of endoscopic therapy for cancer bleeding in patients with unresectable gastric cancer. J Gastroenterol Hepatol 28: 1489-1495, 2013.

23. Crosta C, Spaggiari L, De Stefano A, Fiori G, Ravizza D and Pastorino U: Endoscopic argon plasma coagulation for palliative treatment of malignant airway obstructions: early results in 47 cases. Lung Cancer 33: 75-80, 2001.

24. Heller SJ, Tokar JL, Nguyen MT, Haluszka 0 and Weinberg DS: Management of bleeding GI tumors. Gastrointest Endosc 72: 817-824, 2010.

25. Pereira $\mathrm{J}$ and Phan T: Management of bleeding in patients with advanced cancer. Oncologist 9: 561570, 2004.

26. An Y, Bai Z, Xu X, et al.: No Benefit of Hemostatic Drugs on Acute Upper Gastrointestinal Bleeding in Cirrhosis. Biomed Res Int 2020: 4097170, 2020.

\section{Figures}


5

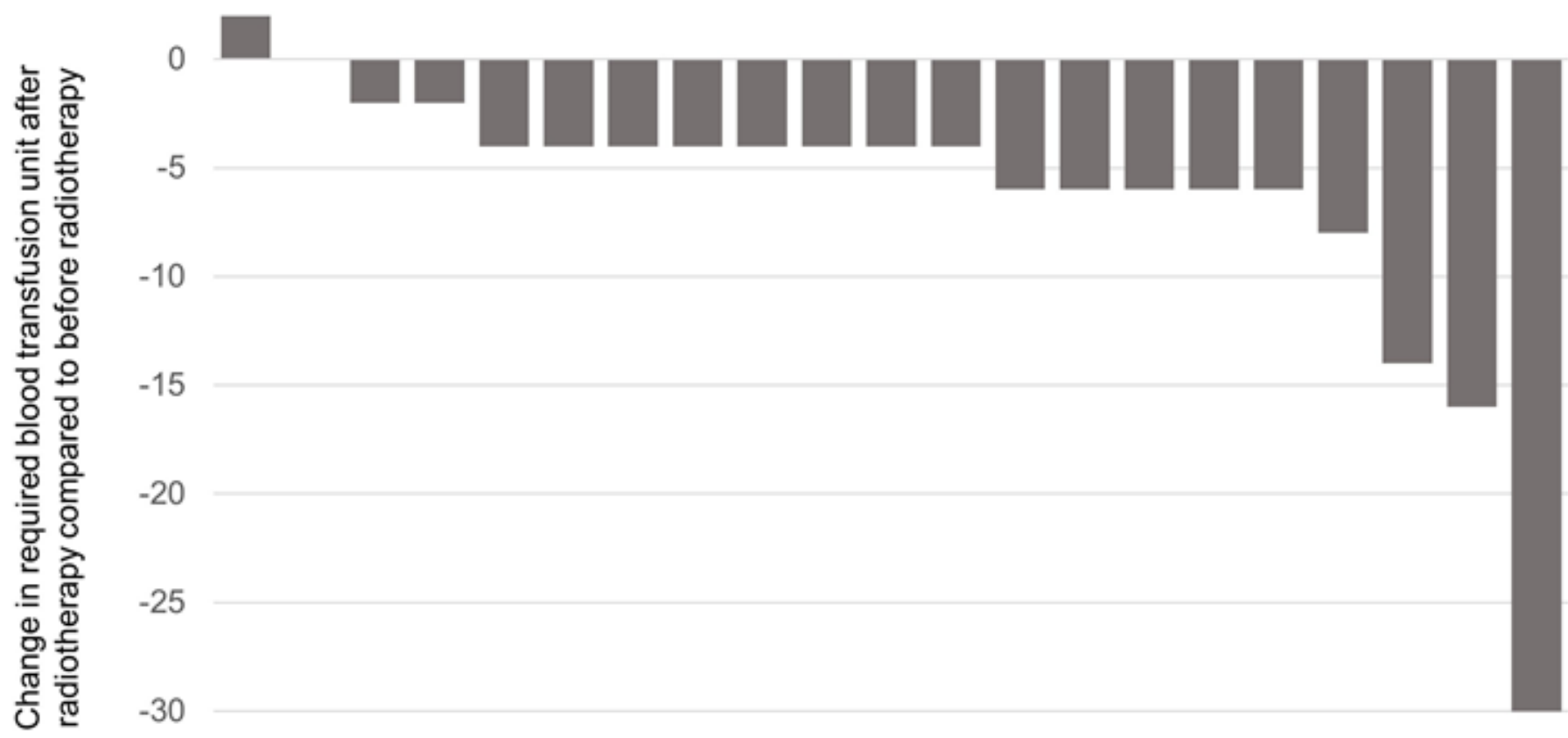

$-35$

Figure 1

Waterfall plot showing the change from the required number of red blood cell transfusion units before radiotherapy to that after radiotherapy in all 21 patients. 


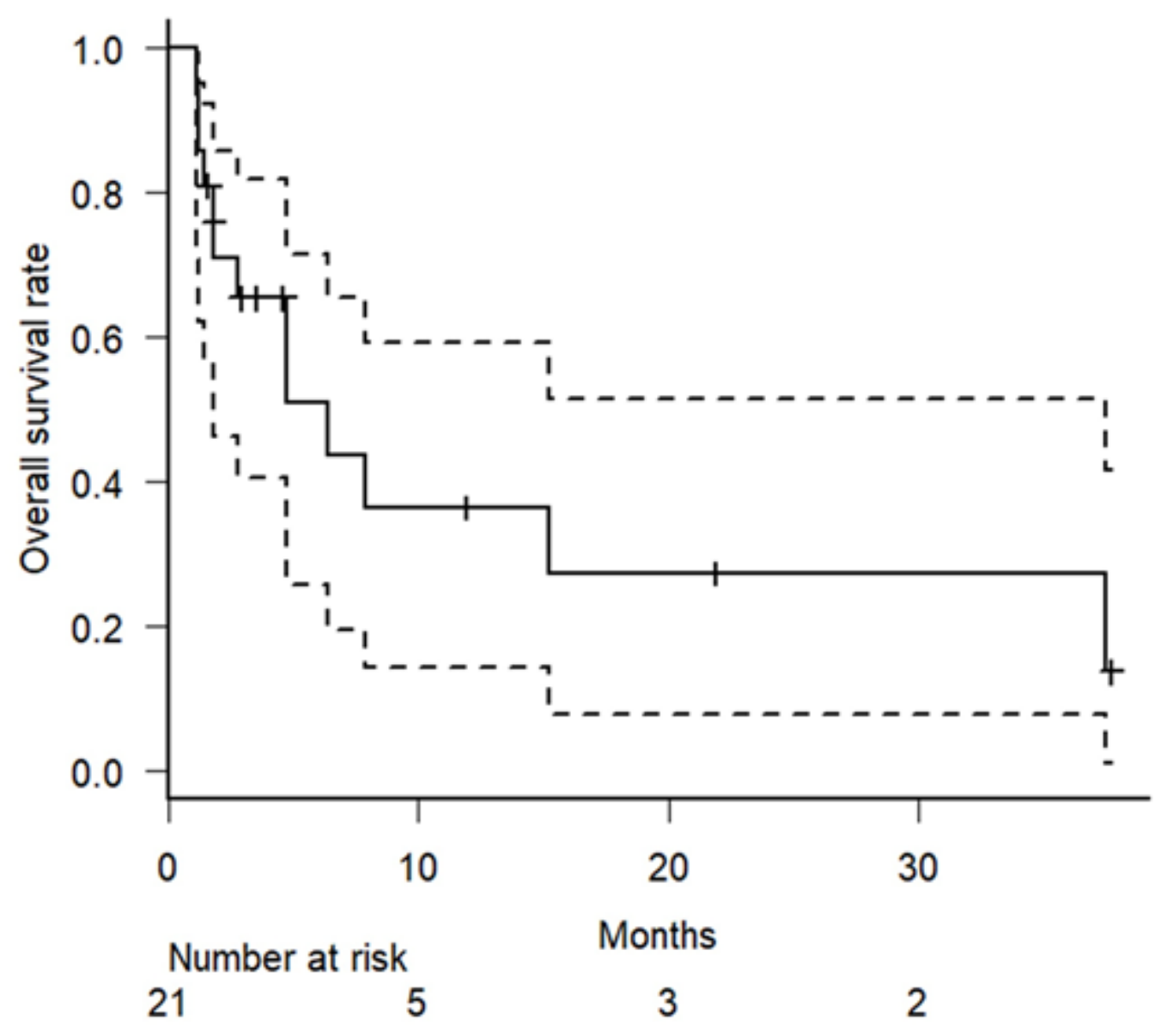

Figure 2

Kaplan-Meier plot depicting the overall survival rate in all 21 patients 


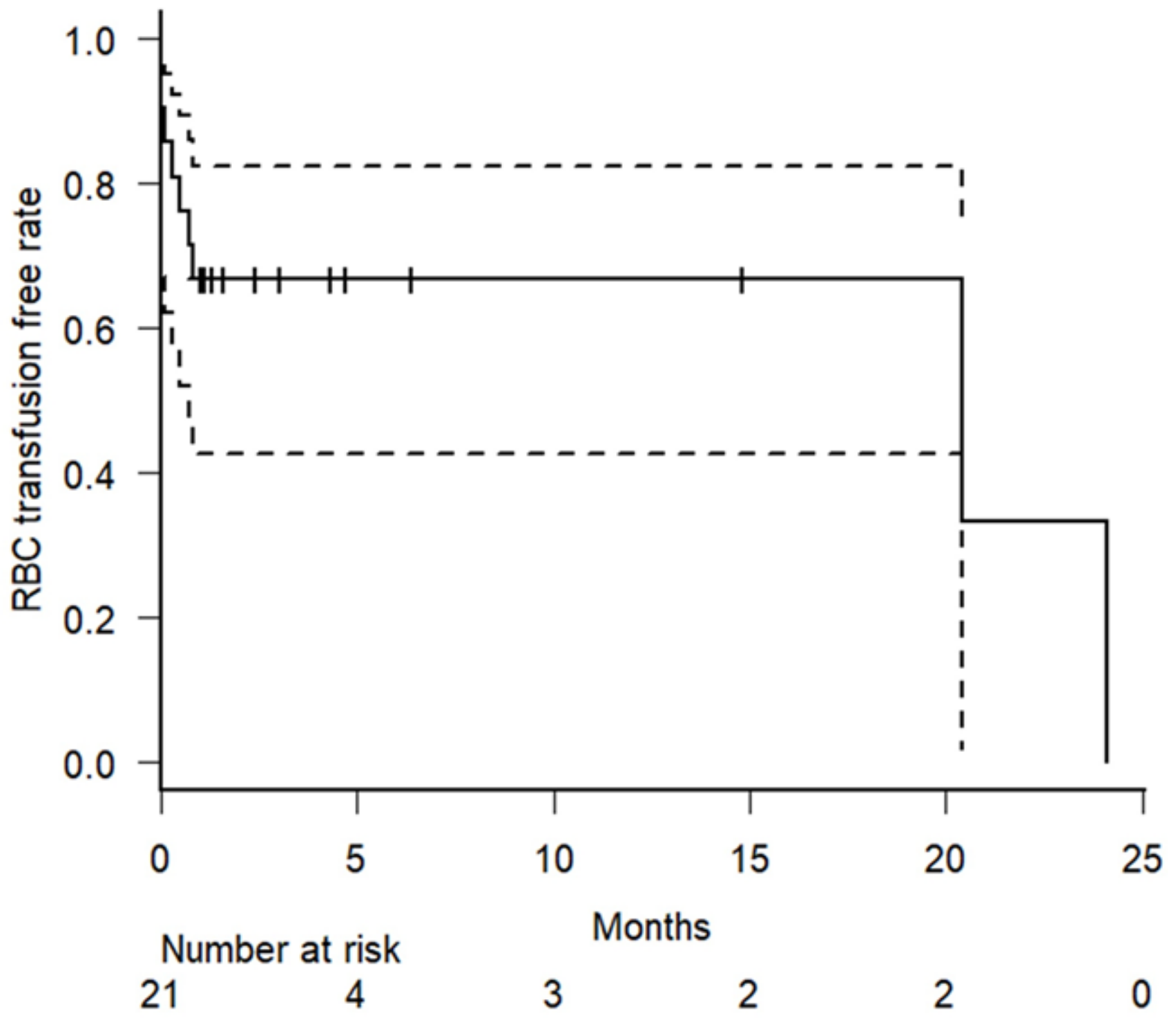

Figure 3

Kaplan-Meier plot depicting the red blood cell (RBC) transfusion-free rate in all 21 patients 